\title{
Effects of surface energy anisotropy on void evolution during irradiation: a phase-field
} model

\author{
W.B. Liu ${ }^{1,2, *}$, N. Wang ${ }^{2}$, Y.Z. Ji ${ }^{2}$, P.C. Song ${ }^{2,3}$, C. Zhang ${ }^{3}$, Z.G. Yang ${ }^{3}$, L.Q. Chen ${ }^{2,3}$ \\ ${ }^{1}$ Department of Nuclear Science and Technology, Xi'an Jiaotong University, Xi'an 710049, \\ China \\ ${ }^{2}$ Department of Materials Science and Engineering, The Pennsylvania State University, \\ University Park, PA 16802, USA \\ ${ }^{3}$ Key Laboratory of Advanced Materials of Ministry of Education, School of Materials \\ Science and Engineering, Tsinghua University, Beijing 100084, China
}

*Author to whom correspondence should be addressed. Email: liuwenbo@xjtu.edu.cn

\begin{abstract}
A phase-field model is employed to investigate the effects of surface energy anisotropy on void evolution during irradiation. By incorporating a simple orientation dependent surface energy with sharp cusps on given crystallographic orientations, experimentally observed void shape with facets and rounded corners is captured. When applied to polycrystalline materials, grain dependent void morphologies are predicted, and the simulation results are qualitatively similar to reported void morphologies in irradiated copper. In addition, the formation of void denuded zones and vacancy depleted zones adjacent to the grain boundaries (GBs) in bicrystalline and polycrystalline structures are studied.
\end{abstract}

Keywords: Surface energy anisotropy; Void growth; Ion irradiation; Phase-field simulation 


\section{Introduction}

Effect of Helium $(\mathrm{He})$ ion irradiation in metals has been the subject of extensive research in the past several decades $[1,2]$. Void and bubble are commonly observed in related experiments. It is reported that an irradiation induced void in a metal is an empty space encased by metallic surfaces [3, 4], while bubbles are typically pressurized helium-filled pores with diameter less than $\sim 10 \mathrm{~nm}[4,5]$. However, voids are the main causes for irradiation induced swelling and embrittlement, while the effect of bubbles on swelling and embrittlement is comparatively small or, in some cases, even beneficial [6]. Moreover, the void morphology exhibits facets that correspond to close-packed planes of the host lattice, while the shape of bubbles is generally spherical [4]. Therefore, the lattice orientation is an important factor for void morphology and evolution during irradiation.

The formation, growth and coarsening of cavities in metals during irradiation are important factors controlling the void swelling, blistering and flacking of nuclear materials [7-9], and voids usually exhibit a well-defined, non-spherical shape [10]. The average size of the voids, which is a key parameter in existing models to describe the irradiation-induced swelling and change of other mechanical properties, is therefore kind of misleading due to the non-spherical nature of voids. Consequently, a more accurate measure of void shape and study for the mechanisms of void shape change is an important prerequisite for a more accurate estimation of the change of mechanical properties of the material during irradiation. There are several influencing factors for the void shape development and its change during irradiation, including surface energy anisotropy, growth anisotropy of void surfaces and a preferential adsorption of atoms on certain surfaces of voids during irradiation [10]. Moreover, 
the shape of voids usually changes with the change of irradiation temperature and/or dose [7, 8], which makes the situation even more complicated. For example, in nickel irradiated by 20 $\mathrm{keV}$ helium ions at $700{ }^{\circ} \mathrm{C}$, the cavities were first in the form of spheres, and then changed to cubes bounded by $\{100\}$ planes and finally changed to cubo-octahedra by the growth of $\{111\}$ facets from cube corners [8]. In this example, there is a clear correlation between the void shape and surface energy anisotropy, and we believe the surface energy anisotropy of voids is an important factor for the development and evolution of void shape during irradiation at given temperatures and irradiation dose.

In the past two decades, many experiments have been devoted to investigating the evolution of void morphologies during irradiation or post-irradiation annealing process in various materials [11-18]. Void-denuded zones were observed along grain boundaries (GBs) and incoherent twin boundaries in copper $(\mathrm{Cu})[11,12]$, nickel (Ni) [13], vanadium (V) [14] and austenitic stainless steel [15] when irradiated at high temperatures. Voids formed during implantation at high temperatures had cubic shapes in $\mathrm{Ni} \mathrm{[16]} \mathrm{and} \mathrm{niobium} \mathrm{(Nb)} \mathrm{[17].}$ Moreover, observations of micro-cavities in $\mathrm{Ni}$ during irradiation and post-irradiation annealing process showed that the shape of voids changed with increased annealing time or annealing temperature $[7,8]$. Theoretically, it is well-known that the shapes of pores or cavities in metals may be determined either by thermodynamic reduction of surface energy or by kinetic considerations governed by interfacial reactions [18]. Therefore, for the slow growing voids in irradiated materials, surface energy and its associated anisotropy may play a key role in determining void morphology. However, a systematic study on the void shapes considering the irradiation dose, temperatures and surface energy anisotropy is still missing 
$[8]$.

Phase-field (PF) simulation is a powerful method in capturing the morphological evolution of interfaces in various materials science processes $[19,20]$. It has been successfully applied to the simulation of nucleation and growth process of bubbles and voids during irradiation [21-25]. Void lattice formation during irradiation [26] and bubble evolution under post-irradiation annealing [27] have also been studied using this method.

It is well known that surface energy anisotropy can be described by a polar plot of the surface energy (also known as the $\gamma$ plot) as a function of local surface orientation. The equilibrium surface shape can be constructed based on the $\gamma$ plot through the Wulff construction $[28,29]$. In previous PF models, surface energy anisotropy was first introduced in dendrite solidification works [30-32] and was also widely used in the study of solid-state phase transformations [33]. However, the effect of surface energy anisotropy on void morphology has not been considered in those PF irradiation models despite its importance in determining void shapes.

While void swelling in irradiated materials involves two steps, void nucleation and void growth [34], the nucleation stage, which depends exponentially on the height of the activation energy barrier, has little impact on the equilibrium shape of the voids, which is mainly obtained during the growth stage. Hence, in the modeling of void swelling in irradiated materials, void nucleation and growth are usually treated as two separate processes $[35,36]$. In the present work, we focus on the void growth behavior, especially the surface energy governed void morphology evolution during irradiation. The surface energy anisotropy is 
incorporated into a PF void growth model under irradiation, a spinodal mechanism is used to spread initial nuclei in numerically, and the effect of surface energy anisotropy on the void morphology is studied in both single crystal and polycrystalline scenarios [21, 22, 25]. The model can be used to interpret and predict the void shape evolution during irradiation based on the orientation dependent surface energy and grain orientation.

\section{PF model}

As mentioned earlier, we assume that the void nucleation and growth stages are separated in time and we focus on the growth stage which begins when the void embryos are established. In order to describe void growth in the metal matrix during irradiation, two field variables are used: a vacancy concentration field $c_{v}(\mathbf{r}, t)$ and a phase field $\eta(\mathbf{r}, t)$. The vacancy concentration $c_{v}(\mathbf{r}, t)$ is defined as the volume fraction of vacancy with respect to the total volume of vacancy and host materials in each lattice site. Here, we use the same definitions as in the works of Rokkam et al [37, 38]: the void phase is characterized by $c_{v}=1.0$ and $\eta=1$, while the matrix phase has $c_{v}=c_{v}^{e q}$ and $\eta=0$. Here $c_{v}^{e q}$ is the equilibrium vacancy concentration in the matrix. Both the concentration and the phase field vary smoothly across the matrix-void interface.

The total free energy of the heterogeneous materials in the present PF model includes the free energy of the void phases, the free energy of matrix phases and the free energy of the interfaces. Based on the definition of free energy in a non-uniform material [39] and the former PF model by Rokkam and co-workers [37, 38], the total free energy, which is a function of the phase field and the vacancy concentration, is written as: 


$$
F=\int_{V}\left[\frac{1}{\Omega} h(\eta) f_{v}^{m}\left(c_{v}\right)+\omega_{0} \frac{k_{B} T}{\Omega} g\left(c_{v}, \eta\right)+\frac{\kappa_{\eta}(\theta)}{2}|\nabla \eta|^{2}+\frac{\kappa_{c}}{2}|\nabla c|^{2}\right] d V
$$

where $\Omega$ is the atomic volume, $\omega_{0}$ and $\kappa_{c}$ are constant coefficient, $T$ is the absolute temperature, $k_{B}$ is the Boltzmann's constant, and $\kappa_{\eta}(\theta)$ is an orientation dependent coefficient that is used to incorporate the surface energy anisotropy. The first two terms on the right hand side (rhs) of Eq. (1) correspond to the bulk energy of the system, and the two gradient terms correspond to the contributions of spatial variation of the field variables, i.e. the interface. The free energy contribution from the vacancy concentration in the matrix $f_{v}^{m}$ in Eq. (1) is:

$$
f_{v}^{m}=c_{v}\left(\Delta E_{v}^{f}-T \Delta S_{v}^{f}\right)+k_{B} T\left[c_{v} \ln \left(c_{v}\right)+\left(1-c_{v}\right) \ln \left(1-c_{v}\right)\right]
$$

where $\Delta E_{v}^{f}$ is the vacancy formation enthalpy, and $\Delta S_{v}^{f}$ is the vacancy formation entropy. The function $h(\eta)$ in Eq. (1) is defined as $h(\eta)=(\eta-1)^{2}(\eta+1)^{2}$ [34]. In the matrix phase $h(\eta)=1$ and it changes smoothly from 1 to 0 at the matrix-void interface. Thus $h(\eta) f_{v}^{m}$ in Eq. (1) gives the correct bulk energy density for both the matrix and the void phase. The potential term $g\left(c_{v}, \eta\right)$ is

$$
g\left(c_{v}, \eta\right)=B_{1}\left(c_{v}-1\right)^{2} \eta^{2}+B_{2}\left(c_{v}-c_{v}^{e q}\right)^{2}(\eta-1)^{2}
$$

where $B_{1}$ and $B_{2}$ are constants, and $c_{v}^{e q}$ is the thermal equilibrium vacancy concentration, with the expression $c_{v}^{e q} \approx \exp \left(-\Delta E_{v}^{f} / k_{B} T\right)$. The evolution equations of the phase field and the concentration field can be written in the form of:

$$
\frac{\partial \eta(\mathbf{r}, t)}{\partial t}=-L_{\eta} \frac{\delta F}{\delta \eta}+\varsigma(\mathbf{r}, t)+P_{v}(\mathbf{r}, t)
$$




$$
\frac{\partial c_{v}(\mathbf{r}, t)}{\partial t}=\nabla \cdot\left(M_{v} \nabla \frac{\delta F}{\delta c_{v}}\right)+\xi(\mathbf{r}, t)+P_{v}(\mathbf{r}, t)-S_{v}^{G B}(1-\Phi)\left(c_{v}-c_{v}^{e q}\right)
$$

where $L_{\eta}$ and $M_{v}$ are the kinetic coefficients related to interface mobility and vacancy diffusivity, respectively, and we use $M_{v}=\Omega D_{v} /\left(k_{B} T\right)$, where $D_{v}$ is the diffusivity of the vacancies; $\varsigma(\mathbf{r}, t)$ and $\xi(\mathbf{r}, t)$ are thermal noise terms; $\quad P_{v}(\mathbf{r}, t)$ is a source term related to the irradiation conditions and acts as a vacancy source during irradiation (see [21] for a detailed description of $\left.P_{v}\right)$. If $\eta \geq 0.8$ or $R_{1}>P_{\text {casc }}$, then $P_{v}(\mathbf{r}, t)=R_{2} V_{G}$; in all other cases, $P_{v}(\mathbf{r}, t)=0$. Here $R_{1}$ and $R_{2}$ are random numbers produced uniformly between zero and one at each time step and at each grid point, and $P_{\text {casc }}$ is the probability of a cascade occurring in a unit volume per unit time [21]. It is worth noting that $P_{\text {casc }}$ has units of displacements per atom (dpa) per time. The last term on the rhs of Eq. (5) incorporates the vacancy sink effect of GBs [38]. $S_{v}^{G B}$ in Eq. (5) is a constant, and the parameter $\Phi$ is a quantity that distinguishes the GB regions from the bulk grains [38]. Using the grain orientation parameter from PF grain growth model $\phi_{i}$ [40-42], which maps the $\mathrm{i}$-th grain to a smooth field, the parameter $\Phi$ can be defined as $\Phi=\sum_{i=1}^{p} \phi_{i}^{2}$. Based on this definition, it is easy to see that $\Phi$ is equal to 1.0 in the grain interiors while much smaller than 1.0 near the GBs and triple junctions. Thus the last term in the rhs of Eq. (5) acts as an effective sink that takes the vacancy concentration from the diffusion equation near the GBs. The functional derivatives in Eqs. (4) and (5) are given as

$$
\frac{\delta F}{\delta \eta}=h^{\prime}(\eta) \frac{1}{\Omega} f_{v}^{m}\left(c_{v}\right)+\omega_{0} \frac{k_{B} T}{\Omega} \frac{\partial g\left(c_{v}, \eta\right)}{\partial \eta}+\frac{\delta}{\delta \eta}\left(\int_{V} \frac{\kappa_{\eta}(\theta)}{2}|\nabla \eta|^{2} d V\right)
$$




$$
\frac{\delta F}{\delta c_{v}}=h(\eta) \frac{1}{\Omega} \frac{\partial f_{v}^{m}\left(c_{v}\right)}{\partial c_{v}}+\omega_{0} \frac{k_{B} T}{\Omega} \frac{\partial g\left(c_{v}, \eta\right)}{\partial c_{v}}-\kappa_{c} \nabla^{2} c_{v}
$$

where $h^{\prime}(\eta)$ in Eq. (6) is the derivative of $h(\eta)$ with respect to $\eta$. After combining all the equations above, the final form of the kinetic equations can be rewritten as:

$$
\begin{aligned}
\frac{\partial \eta(\mathbf{r}, t)}{\partial t}=- & L_{\eta} \frac{\omega_{0} k_{B} T}{\Omega}\left[h^{\prime}(\eta) \frac{1}{\omega_{0} k_{B} T} f_{v}^{m}\left(c_{v}\right)+\frac{\partial g\left(c_{v}, \eta\right)}{\partial \eta}+\frac{\Omega}{\omega_{0} k_{B} T} \frac{\delta}{\delta \eta}\left(\int_{V} \frac{\kappa_{\eta}(\theta)}{2}|\nabla \eta|^{2} d V\right)\right] \\
& +\varsigma(\mathbf{r}, t)+P_{v}(\mathbf{r}, t) \\
\frac{\partial c_{v}(\mathbf{r}, t)}{\partial t}=\nabla & \cdot\left[M_{v} \frac{\omega_{0} k_{B} T}{\Omega} \nabla\left[h(\eta) \frac{1}{\omega_{0} k_{B} T} \frac{\partial f_{v}^{m}\left(c_{v}\right)}{\partial c_{v}}+\frac{\partial g\left(c_{v}, \eta\right)}{\partial c_{v}}-\frac{\Omega}{\omega_{0} k_{B} T} \kappa_{c} \nabla^{2} c_{v}\right]\right] \\
& +\xi(\mathbf{r}, t)+P_{v}(\mathbf{r}, t)-S_{v}^{G B}(1-\Phi)\left(c_{v}-c_{v}^{e q}\right)
\end{aligned}
$$

To incorporate the surface energy anisotropy in this PF model, the orientation dependent gradient coefficient $\frac{\Omega}{\omega_{0} k_{B} T} \kappa_{\eta}(\theta)$ is rewritten as:

$$
\sqrt{\frac{\Omega}{\omega_{0} k_{B} T} \kappa_{\eta}(\theta)}=W(\theta)=W_{0} f\left(\theta-\theta_{0}\right)
$$

where $\theta$ is the surface orientation angle defined between the normal direction of the interface and some fixed crystalline axis given by $\theta_{0}$, and $f\left(\theta-\theta_{0}\right)$ is a orientation dependent function. Here the angle $\theta$ is given by $\theta=\arctan \left(\eta_{y} / \eta_{x}\right)$, where $\eta_{y}$ and $\eta_{x}$ are the derivatives of $\eta$ with respect to y and $\mathrm{x}$ respectively [33]. To reproduce the square void shape observed in $\mathrm{Cu}$ and $\mathrm{Ni}$ [11-13], a simple orientation function

$$
f\left(\theta-\theta_{0}\right)=B_{0}+\delta\left(\left|\sin \left(\theta-\theta_{0}\right)\right|+\left|\cos \left(\theta-\theta_{0}\right)\right|\right)
$$

is used since such a function gives a square equilibrium shape in Wulff construction. In Eq. 11, $B_{0}$ and $\delta$ are constants, and can both be identified based on the real surface energy of the designated materials. The $\gamma$ plot and Wulff construction with $B_{0}=1.0, \theta_{0}=0$ and $\delta=1.0$ are 
shown in Fig. 1. It can be seen that in the $\gamma$ plot there are 4 minimum points (when $\theta=0^{\circ}$, $90^{\circ}, 180^{\circ}, 270^{\circ}$, and corresponding to $<10>$ directions in 2 dimensions (2D)) and 4 maximum points (when $\theta=45^{\circ}, 135^{\circ}, 225^{\circ}, 315^{\circ}$, and corresponding to $\langle 11>$ directions in 2D), respectively. The final void shape with facets and rounded corners is determined by the 4 minimum values through Wulff construction.

Compared with previous PF void growth models [21, 22], interstitials are not included in this work. Since the purpose of this work is to demonstrate the effects of surface energy anisotropy on the void morphology evolution during irradiation, the impact of this simplification is not a major concern. The thermal noise terms are also ignored in this work.

In numerical implementation, equations are solved in reduced form with non-dimensionalized length $\tilde{x}=x / W_{0}$ and time $\tilde{t}=t / \tau$ where $\tau=W_{0}^{2} / D_{v}$. The simulation temperature is $500 \mathrm{~K}$ (Below the melting point of pure $\mathrm{Cu}$ : $1357.77 \mathrm{~K}$ ). The vacancy formation energy $\Delta E_{v}^{f}$ in $\mathrm{Cu}$ is set to $1.03 \mathrm{eV}$ [43], and the corresponding thermal equilibrium vacancy concentration at $500 \mathrm{~K}$ is $4.19 \times 10^{-11}$. The length scale is taken as $W_{0}=$ $10 \mathrm{~nm}$, and the computational grid spacing is also equal to $W_{0}$. The vacancy diffusivity in $\mathrm{Cu}$ at $500 \mathrm{~K}$ is taken as $D_{\mathrm{v}}=757 \mathrm{~nm}^{2} / \mathrm{s}[43,44]$, and the time scale is then taken as $\tau=W_{0}^{2} / D_{v}=0.132 s$. The displacement rate in the present simulation is taken as $3.78 \times 10^{-3}$ dpa/s. After normalization, the evolution Eqs. (8) and (9) become:

$$
\begin{aligned}
\frac{\partial \eta \tilde{\mathbf{r}, \tilde{t})}}{\partial \tilde{t}}= & -\tilde{L}_{\eta}\left[h^{\prime}(\eta) \tilde{f}_{v}^{m}\left(c_{v}\right)+2 B_{1}\left(c_{v}-1\right)^{2} \eta+2 B_{2}\left(c_{v}-c_{v}^{e q}\right)^{2}(\eta-1)+\tilde{\nabla} \cdot\left[W(\theta)^{2} \tilde{\nabla} \eta\right]\right. \\
& \left.-\partial_{x}\left[W(\theta) W^{\prime}(\theta) \partial_{y} \eta\right]+\partial_{y}\left[W(\theta) W^{\prime}(\theta) \partial_{x} \eta\right]\right]+\varsigma(\tilde{\mathbf{r}}, \tilde{t})+P_{v}(\tilde{\mathbf{r}}, \tilde{t})
\end{aligned}
$$




$$
\begin{aligned}
\frac{\partial c_{v}(\tilde{\mathbf{r}}, \tilde{t})}{\partial \tilde{t}}= & \tilde{\nabla} \cdot\left(\tilde { M } _ { v } \tilde { \nabla } \left[h(\eta)\left(\tilde{E}_{v}^{f}+\ln \left(c_{v}\right)-\ln \left(1-c_{v}\right)\right)-\tilde{\kappa}_{c} \tilde{\nabla}^{2} c_{v}+2 B_{1}\left(c_{v}-1\right) \eta^{2}\right.\right. \\
& \left.\left.-2 B_{2}\left(c_{v}-c_{v}^{e q}\right)(\eta-1)^{2}\right]\right)+\xi(\tilde{\mathbf{r}}, \tilde{t})+P_{v}(\tilde{\mathbf{r}}, \tilde{t})-S_{v}^{G B}(1-\Phi)\left(c_{v}-c_{v}^{e q}\right)
\end{aligned}
$$

where $\tilde{\nabla}$ is the dimensionless gradient operator, and $\tilde{L}_{\eta}=L_{\eta} \omega_{0} \tau \frac{k_{B} T}{\Omega}$. The function $\tilde{f_{v}^{m}}\left(c_{v}\right)$ is obtained by normalizing $f_{v}^{m}\left(c_{v}\right)$ with respect to $\omega_{0} k_{B} T$. Likewise, the parameter $\Delta \tilde{E_{v}^{f}}$ is obtained by normalizing $\Delta E_{v}^{f}$, with respect to $\omega_{0} k_{B} T$. The mobility of the vacancies in the dimensionless form can be expressed as $\tilde{M}_{v}=M_{v} \omega_{0} \frac{k_{B} T}{\Omega} \frac{W_{0}^{2}}{\tau}$, and the dimensionless gradient energy coefficient $\tilde{\kappa}_{c}$ in Eq. (13) is $\tilde{\kappa}_{c}=\frac{\Omega}{\omega_{0} k_{B} T W_{0}^{2}} \kappa_{c}$. The derivation of Eq. (12) is given in Appendix A. In the present work, Eqs. (12) and (13) are solved using simple forward Euler time integration and finite-difference space discretization on a uniform grid with periodic boundary conditions. The following values of parameter are used for the calculation: $B_{1}=1.0, B_{2}=1.0, \tilde{M}_{v}=1.0, \tilde{L}_{\eta}=1.0, \tilde{\kappa}_{c}=1.0$. For the simulation of morphology evolution of a single void, the simulation domain was divided into $128 \times 128$ grid points, and in all other scenarios, the domain was divided into $512 \times 512$ grid points.

\section{Results and discussion}

\subsection{Morphology evolution of a single void}

It is generally accepted that a net absorption of vacancies to the surface of a void will result in void growth. To demonstrate the effect of surface energy anisotropy on the void shape evolution, we consider the growth of a single void in the matrix due to supersaturated vacancy concentration. We initialize the system to contain a circular void with radius $\tilde{r}=5$ 
at the center of the domain. In the surrounding solid region, a uniform supersaturation of vacancies is initialized as $c_{v}=100 c_{v}^{e q}$, which is similar to the assumption made in previous models $[37,38]$. The high vacancy concentration in this section can be viewed as a way to obtain fast numerical results. Moreover, in the present section, the shape of the void has nothing to do with the vacancy concentration in the matrix surrounding the void since there is no irradiation ( $\mathrm{P}_{\mathrm{v}}$ in Eq. 4 and 5 is turned off). It is reported that the surface energies of $\{100\}$ and $\{110\}$ planes in $\mathrm{Cu}$ are $2.090 \mathrm{~J} / \mathrm{m}^{2}$ and $2.310 \mathrm{~J} / \mathrm{m}^{2}$, respectively [45]. In the present work, we consider a simple form of $W(\theta)=\gamma_{0} f(\theta)$ and $f(\theta)=1.0+0.5(|\sin (\theta)|+|\cos (\theta)|)$ with $\gamma_{0}=1.33 \mathrm{~J} / \mathrm{m}^{2}$, and the minimum and maximum values of $W(\theta)$ are 1.995 (when $\theta=0^{\circ}, 90^{\circ}, 180^{\circ}, 270^{\circ}$, and corresponding to the $<10>$ directions in 2D) and 2.270 (when $\theta=45^{\circ}, 135^{\circ}, 225^{\circ}, 315^{\circ}$, and corresponding to the $<11>$ directions in 2D), respectively. The coefficient $S_{v}^{G B}$ is set as 0.0 since there are no GBs.

The evolutions of $\eta(\tilde{\mathbf{r}}, \tilde{t})$ and $c_{v}(\tilde{\mathbf{r}}, \tilde{t})$ are shown in Fig.2. It can be seen that significant void growth happens because of the absorption of vacancies from the surrounding solid matrix. Due to the effect of surface energy anisotropy, faceted void surface connected with rounded corners is developed as the void grows in size, and such a void shape is similar to that observed in irradiated $\mathrm{Cu}[11]$.

Transmission electron microscope (TEM) observations show that voids formed during irradiation are usually cubes bounded by the $\{100\}$ facets $[8,14]$, or truncated octahedra bounded by the $\{111\}$ planes [11], or cuboctahedra bounded by the $\{100\}$ and the $\{111\}$ facets [8]. Thus, voids in different grains usually show the same shape, but have different 
"orientations" due to the different orientations of grains [12]. In order to investigate the effect of the underlying lattice orientation difference in polycrystalline materials on the void morphology, change of the crystal axis angle $\theta_{0}$ for different grains is considered. Here, we demonstrate the change of the void morphology as a result of different grain lattice orientation. By introducing a rotation angle of $\theta_{0}=45^{\circ}$ to the crystal axis, void shape and vacancy concentration are developed following the rotated axis as shown in Fig. 3. It can be seen that the angle between the $\langle 10\rangle$ directions of the new grain and the horizontal axis is $45^{\circ}$, and the <11> directions, with the maximum value of surface energy, are coincident with the horizontal axis. As a result, the final void shape is also rotated $45^{\circ}$ compared to that in Fig. 1.

\subsection{Morphology evolution in polycrystals}

As mentioned in Section 3.1, it has been observed experimentally that voids in different grains have different orientations although all the voids have approximately the same shape due to the same irradiation condition $[8,14,11]$. Due to sinks of irradiation-induced point defects by GBs, the void denuded zone adjacent to the GBs has been observed in various irradiated materials, including $\mathrm{Cu}[11,12], \mathrm{Ni}$ [13] and V [14].

In this section, we employ the current PF model to simulate the void morphology evolution in a bicrystal geometry with a flat GB in the middle and an additional GB at the end of the simulation box due to the periodic boundary condition. The system is initialized to contain uniform vacancies with $c_{v}=c_{v}^{e q}$, corresponding to the equilibrium conditions. The evolution of the void structures is governed by Eqs. (12) and (13) with the irradiation source turned on. 
The void morphology evolution of the bicrystal geometry is shown in Fig. 4, with $\theta_{0}=45^{\circ}$ for the left grain and $\theta_{0}=22.5^{\circ}$ for the right grain. It can be seen that the shapes of the void display facets connected by rounded corners in both grains, but the orientations of voids in the left grain and the right grain are different, which are similar to the experimental observation in irradiated $\mathrm{Cu}[11]$. However, voids in the same grain have the same orientation due to the fact that all the voids in the same grain are developed based on the same underlying lattice orientation. After $\sim 525$ simulation time (Fig. 4d), the void depleted zone adjacent to the GBs is observed due to the sink effect of the GBs. With increasing time, the average void size increases and the void number density decreases due to coarsening [46]. In addition, depleted regions of vacancies are observed near the void surface, since the void surface also acts as a vacancy sink.

With the above analysis of void nucleation and growth in a bicrystal structure, we now focus on void nucleation and growth in polycrystalline microstructures. In the PF model of grain growth [41, 42], polycrystalline microstructure is produced using a set of continuous, non-conserved phase fields. Each of these phase fields tracks the evolution of a single grain with specific lattice orientation. In this work, we use the simulation results from the PF grain growth model [40-42] as the initial microstructure to investigate the evolution of void morphology in polycrystalline materials. Since we mainly focus on the void morphology in polycrystalline structures, we ignore the possible grain growth during the irradiation process. Moreover, we incorporate different lattice orientations in the model through the angle $\theta_{0}$ defined in Eq. (11) based on the polycrystalline grain structure. The system is also initialized to contain a uniform vacancy distribution with $c_{v}=c_{v}^{e q}$, corresponding to the 
equilibrium conditions, and the evolution of phase field and vacancy concentration is governed by Eqs. (12) and (13) with the irradiation source turned on.

The nucleation and growth of void in crystalline materials due to the ongoing production of vacancies are shown in Fig. 5. It can be clearly seen that, with the consideration of surface energy anisotropy, voids in different grains have different orientations, and voids in the same grain have the same orientation, which is quite similar to the void morphology in irradiated polycrystalline $\mathrm{Cu}[12]$. In addition, with increasing simulation time, vacancy depleted zones and/or void denuded zones surrounding the GBs are observed due to the annihilation of irradiation-induced point defects, and the void surfaces also act as sinks for vacancies, which is the similar to the experimental observation $[11,12]$ and previous simulation works [38].

As mentioned earlier, a void is essentially an empty cavity, while a bubble is usually filled with gas atoms under high pressure. However, voids usually have a profound influence on materials properties because solids undergo volumetric swelling when voids form and grow [34]. The size of void can vary from 2 to several tens of nanometers with a number density of $10^{21} \sim 10^{22} \mathrm{~m}^{-2}$, and thus can cause tens of percent of volume expansion. As discussed earlier, experimental results show that voids in $\mathrm{Cu}$ and $\mathrm{Ni}$ usually have polyhedron shapes, and the surrounding surfaces are planes with low Miller index of the matrix, and similar results are also found in irradiated austenite steel and ferrite/martensite steel [34]. Moreover, when the irradiation dose or temperature is high, the void shapes are usually squares.

Surface energy is an important factor which influences the void morphology evolution 
during irradiation since void is an empty cavity. The swelling, which is an important property for irradiated materials, can be calculated based on the void growth behavior. Hence, it is critical to correctly simulate the growth behavior of polyhedron shaped void during irradiation. The present model can interpret and predict the shape evolution and growth behavior with the consideration of surface energy anisotropy, and the model can be extended to be three-dimensional. The model can also handle the calculation of swelling, which is our later work.

\section{Summary}

By introducing surface energy anisotropy and its variation due to the lattice orientation difference in polycrystalline materials, the PF void growth model is successfully extended to simulate the faceted void morphologies that are commonly observed in copper after irradiation. In the simulation of single crystal, void in the shape of facets connected with rounded corners is formed. In the simulations of polycrystalline structures containing a uniform equilibrium vacancy concentration, voids with facets connected with rounded corners are formed after irradiation, and voids in different grains exhibit different orientations based on the underlying lattice orientation of the host grain. In addition, void denuded zones and vacancy depleted zones adjacent to the grain boundaries (GBs) in bicrystalline and polycrystalline structures are formed due to the vacancy annihilation near GBs.

\section{Acknowledgments}

The authors are grateful to Dr. Di Yun for useful discussion and suggestions. This work was 
partially supported by DOE Basic Sciences under the CMCSN Program. Financial support from the Project Funded by China Postdoctoral Science Foundation (no. 2015M582669) and National Basic Research Programs of China (no. 2015GB118001) is also acknowledged. The computer simulations were carried out on the LION clusters at the Pennsylvania State University.

Appendix A. Derivation of the interfacial term in Eq. (12)

Combining Eq. (10) and Eq. (1), we can get:

$$
\begin{aligned}
F & =\int\left[\frac{1}{\Omega} h(\eta) f_{v}^{m}\left(c_{v}\right)+\omega_{0} \frac{k_{B} T}{\Omega} g\left(c_{v}, \eta\right)+\frac{\kappa_{c}}{2}|\nabla c|^{2}+\omega_{0} \frac{k_{B} T}{\Omega} \frac{W(\theta)^{2}}{2}|\nabla \eta|^{2}\right] d v \\
& =\omega_{0} \frac{k_{B} T}{\Omega} \int\left[f_{p}+\frac{W(\theta)^{2}}{2}|\nabla \eta|^{2}\right] d v=\omega_{0} \frac{k_{B} T}{\Omega} \int f_{v} d v
\end{aligned}
$$

where $f_{p}$ is the density of total free energy except the last term in Eq. (A1), $\theta=\arctan \left(\eta_{y} / \eta_{x}\right)$, while $\eta_{y}$ and $\eta_{x}$ are the derivatives of $\eta$ with respect to $\mathrm{y}$ and $\mathrm{x}$ respectively. With these equations in hand, we derive the variational derivative of $F$ as follows:

$$
F=\int\left[f_{p}+\frac{W(\theta)^{2}}{2}|\nabla \eta|^{2}\right] d v=\int f_{v} d v
$$




$$
\begin{aligned}
\frac{\delta F}{\delta \eta} & =\frac{\partial f_{v}}{\partial \eta}-\left[\frac{\partial}{\partial x} \frac{\partial f_{v}}{\partial \eta_{x}}+\frac{\partial}{\partial y} \frac{\partial f_{v}}{\partial \eta_{y}}\right] \\
& =\frac{\partial f_{p}}{\partial \eta}-\left[\frac{\partial}{\partial x} \frac{\partial\left[\frac{W(\theta)^{2}}{2}\left(\eta_{x}^{2}+\eta_{y}^{2}\right)\right]}{\partial \eta_{x}}+\frac{\partial}{\partial y} \frac{\partial\left[\frac{W(\theta)^{2}}{2}\left(\eta_{x}^{2}+\eta_{y}^{2}\right)\right]}{\partial \eta_{y}}\right] \\
& =\frac{\partial f_{p}}{\partial \eta}-\left[\frac{\partial}{\partial x}\left[W(\theta)^{2} \eta_{x}\right]+\frac{\partial}{\partial y}\left[W(\theta)^{2} \eta_{y}\right]\right]-\left[\frac{\partial}{\partial x} \frac{\left[\left(\eta_{x}^{2}+\eta_{y}^{2}\right) \frac{\partial W(\theta)^{2}}{2 \partial \eta_{x}}\right]}{2 \partial \eta_{x}}+\frac{\partial}{\partial y} \frac{\partial\left[\left(\eta_{x}^{2}+\eta_{y}^{2}\right) \frac{\partial W(\theta)^{2}}{2 \partial \eta_{y}}\right]}{2 \partial \eta_{y}}\right] \\
& =\frac{\partial f_{p}}{\partial \eta}-W(\theta)^{2} \nabla^{2} \eta-2 W(\theta) \nabla W(\theta) . \nabla \eta-\left[\frac{\partial}{\partial x} \frac{\left[\left(\eta_{x}^{2}+\eta_{y}^{2}\right) \frac{\partial W(\theta)^{2}}{2 \partial \eta_{x}}\right]}{2 \partial \eta_{x}}+\frac{\partial}{\partial y} \frac{\partial\left[\left(\eta_{x}^{2}+\eta_{y}^{2}\right) \frac{\partial W(\theta)^{2}}{2 \partial \eta_{y}}\right]}{2 \partial \eta_{y}}\right]
\end{aligned}
$$

where

$$
\begin{aligned}
\frac{\partial W(\theta)^{2}}{\partial \eta_{x}} & =2 W(\theta) \frac{\partial W\left(\arctan \left(\eta_{y} / \eta_{x}\right)\right)}{\partial \eta_{x}} \\
& =2 W(\theta) \frac{\partial W(\theta)}{\partial \theta} \frac{\partial \arctan \left(\eta_{y} / \eta_{x}\right)}{\partial\left(\eta_{y} / \eta_{x}\right)} \frac{\partial\left(\eta_{y} / \eta_{x}\right)}{\partial \eta_{x}} \\
& =2 W(\theta) \frac{W^{\prime}(\theta)}{1+\left(\eta_{y} / \eta_{x}\right)^{2}}\left[-\frac{\eta_{y}}{\eta_{x}^{2}}\right] \\
& =-2 W(\theta) W^{\prime}(\theta) \frac{\eta_{y}}{\eta_{x}^{2}+\eta_{y}^{2}}
\end{aligned}
$$

and

$$
\frac{\partial W(\theta)^{2}}{\partial \eta_{y}}=2 W(\theta) W^{\prime}(\theta) \frac{\eta_{x}}{\eta_{x}^{2}+\eta_{y}^{2}}
$$

Therefore, we obtain:

$$
\frac{\delta F}{\delta \eta}=\frac{\partial f_{p}}{\partial \eta}-W(\theta)^{2} \nabla^{2} \eta-2 W(\theta) \nabla W(\theta) \cdot \nabla \eta+\partial_{x}\left[W(\theta) W^{\prime}(\theta) \eta_{y}\right]-\partial_{y}\left[W(\theta) W^{\prime}(\theta) \eta_{x}\right]
$$

Then, we can obtain Eq. (12) by combining Eq. (A6) and Eq. (8).

\section{References}

[1] K. Krishan, Rad. Eff. 66 (1982) 121-155.

[2] H. Ullmaier, Rad. Eff. 78 (1983) 1-10.

[3] Z.H. Xiao, A.A. Semenov, C.H. Woo, S.Q. Shi, J. Nucl. Mater. 439 (2013) 25-32.

[4] S.J. Zinkle (2012) Radiation-Induced Effects on Microstructure. In: R.J.M. Konings (ed.) Comprehensive Nuclear Materials, volume 1, pp. 65-98 
[5] L.K. Mansur, W.A. Coghlan, J. Nucl. Mater. 119 (1983) 1-25.

[6] G.R. Odette, D.T. Hoelzer. JOM 62(2010) 84-92.

[7] K. Niwase, T. Ezawa, T. Tanabe, F.E. Fujita, J. Nucl. Mater. 160 (1988) 229-241.

[8] K. Niwase , T. Ezawa , F.E. Fujita , H. Kusanagi, H. Takaku, Rad. Eff. 106 (1988) 65-76.

[9] J. O. Stiegler, E. E. Bloom, Rad. Eff. 8 (1971) 33-41.

[10] C.W. Chen, Phys. Stat. Sol. (a) 16 (1973) 197-210.

[11] S.J. Zinkle, K. Farrell, J. Nucl. Mater. 168 (1989) 262-267

[12] B.N. Singh, A. Horsewell, D.S. Gelles, F.A. Garner, J. Nucl. Mater. 191 (1992) $1172-1176$

[13] C.W. Chen, R.W. Buttry, Rad. Eff. 56 (1981) 219-228.

[14] M. Cambini, J. Bressers, M. Heerschap, J. Nucl. Mater. 62 (1976) 311-313.

[15] I.S. Kim, J.D. Hunn, N. Hashimoto, D.L. Larson, P.J. Maziasz, K. Miyahara, E.H. Lee, J. Nucl. Mater. 280 (2000) 264-274.

[16] V. Zell, H. Schroeder, H. Trinkaus, J. Nucl. Mater. 212-215 (1994) 358-363.

[17] B.A. Loomis, S.B. Gerber, J. Nucl. Mater. 102 (1981) 154-169.

[18] P.J. Goodhew, Metal Science 15 (1981) 377-385.

[19] L.Q. Chen, Annu. Rev. Mater. Res. 32 (2002) 113-140.

[20] W. J. Boettinger, J. A.Warren, C. Beckermann, A. Karma, Annu. Rev. Mater. Res. 32 (2002) 163-194.

[21] P.C. Millett, A. El-Azab, S. Rokkam, M. Tonks, D. Wolf, Comp. Mater. Sci. 50 (2011) 949-959.

[22] P.C. Millett, A. El-Azab, D. Wolf, Comp. Mater. Sci. 50 (2011) 960-970.

[23] S. Rokkam, A. El-Azab, MRS Proc. - Camb. J. (2011) 1363.

[24] A.A. Semenov, C.H. Woo, Acta Mater. 60 (2012) 6112-6119.

[25] N. Wang, S. Rokkam, T. Hochrainer, M. Pernice, A. El-Aza, Comp. Mater. Sci. 89 (2014) 165-175.

[26] S. Hu, C.H. Henager Jr, J. Nucl. Mater. 394 (2009) 155-159.

[27] Y. Li, S. Hu, R. Montgomery, F. Gao, X. Sun, Nucl. Instrum. Method B 303 (2013) 62-67.

[28] C. Herring, Phys. Rev. 82 (1951) 87.

[29] M.R. Castell, Phys. Rev. B 68 (2003) 235411.

[30] G.B. McFadden, A.A. Wheeler, R.J. Braun, S.R. Coriell, Phys. Rev. E 48 (1993) 2016.

[31] R. Kobayashi, Physica D 63 (1998) 410-423.

[32] J.M. Debierre, A. Karma, F. Celestini, R. Guerin, Phys. Rev. E 68 (2003) 041604.

[33] M. Greenwood, J.J. Hoyt, N. Provatas, Acta Mater. 57 (2009) 2613-2623.

[34] G.S. Was, Fundamentals of Radiation Materials Science (Springer, 2007)

[35] K.C. Russell, Acta Mater. 19 (1971) 753-758.

[36] S.I. Golubov, A.M. Ovcharenko, A.V. Barashev, B.N. Singh, Philos. Mag. A 81 (2001) 643-658.

[37] S. Rokkam, A. El-Azab, P. Millett, D. Wolf, Modelling Simul. Mater. Sci. Eng. 17 (2009) 064002 .

[38] P.C Millett, S. Rokkam, A. El-Azab, M. Tonks, D. Wolf, Modelling Simul. Mater. Sci. Eng. 17 (2009) 064003.

[39] J.W. Cahn, J.E. Hilliard, J. Chem. Phys. 28 (1958) 258-267. 
[40] L.Q. Chen, W. Yang, Phys. Rev. B 50 (1994) 15752.

[41] D. Fan, L.Q. Chen, Acta Mater. 45 (1997) 611-622.

[42] D. Fan, C. Geng, L.Q. Chen, Acta Mater. 45 (1997) 1115-1126.

[43] H. Mehrer, A. Seeger, Phys. Stat. Sol. (b) 35 (1969) 313-328.

[44] P.C. Millett, M. Tonks, Comp. Mater. Sci. 50 (2011) 2044-2050.

[45] H.L. Skriver, N.M. Rosengaard, Phys. Rev. B 46 (1992) 7157.

[46] H. Trinkaus, B.N. Singh, J. Nucl. Mater. 323 (2003) 229-242.

Captions

Fig.1 Typical polar plot of surface free energy for a crystal (a) and the Wulff construction based on it (b), and hence the void shape consisting of four facets joined by smoothly curved regions.

Fig.2 Effects of surface energy anisotropy on the void morphology evolutions of $\eta(\tilde{\mathbf{r}}, \tilde{t})$ and $c_{v}(\tilde{\mathbf{r}}, \tilde{t})$ with an initial supersaturation of vacancies: (a) $\eta(\tilde{\mathbf{r}}, 0)$, (b) $\eta(\tilde{\mathbf{r}}, 45)$, (c) $\eta(\tilde{\mathbf{r}}, 90)$, (d) $c_{v}(\tilde{\mathbf{r}}, 0),(\mathrm{e}) c_{v}(\tilde{\mathbf{r}}, 45),(\mathrm{f}) c_{v}(\tilde{\mathbf{r}}, 90)$. Inset in (a) shows a global coordinate $\left(\theta_{0}=0\right)$.

Fig.3 Effects of surface energy anisotropy on the morphology evolutions with orientation of $\theta_{0}=45^{\circ}$ in Eq. (11): (a) $\gamma$ plot (dashed red line) and inner envelope (full blue line) (b) $\eta(\tilde{\mathbf{r}}, 90)$ and (c) $c_{v}(\tilde{\mathbf{r}}, 90)$. Inset in (b) shows a global coordinate $\left(\theta_{0}=0\right)$.

Fig.4 Void morphology evolutions in a bi-crystal structure under the effect of surface energy anisotropy for different grains: (a)0 dpa, (b) $0.09 \mathrm{dpa}$, (c) $0.18 \mathrm{dpa}$, (d) $0.27 \mathrm{dpa}$, (e) $0.36 \mathrm{dpa}$. This phenomenon has been observed in irradiated copper [11] as shown in (f) (picture taken from [11]). In all the simulations (a), (b), (c), (d) and (e), the crystalline orientation angles $\theta_{0}$ 
are set as $\theta_{0}=45^{\circ}$ for the left grain and $\theta_{0}=22.5^{\circ}$ for the right grain. Inset in (a) shows a global coordinate $\left(\theta_{0}=0\right)$.

Fig.5 Void morphology evolutions in a polycrystalline grain structure under the effect of surface energy anisotropy and stochastic vacancy generation: (a) $0.15 \mathrm{dpa}$, (b) $0.225 \mathrm{dpa}$, (c) 0.3 dpa. Inset in (a) shows a global coordinate $\left(\theta_{0}=0\right)$. 

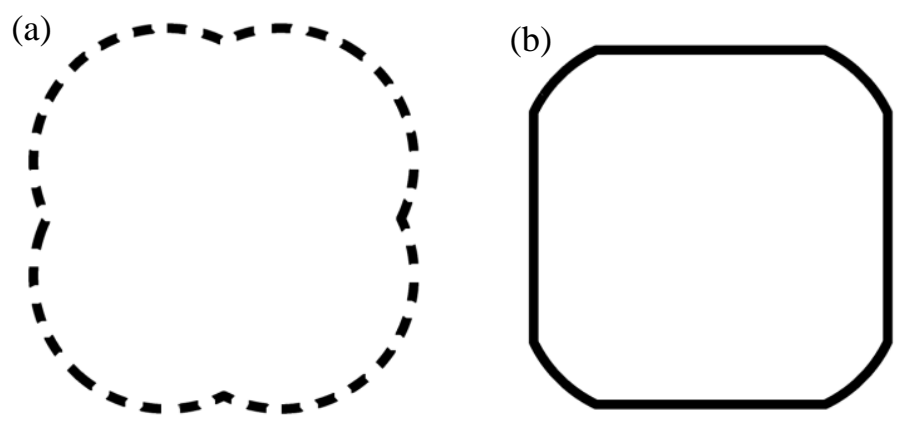

Fig.1 Typical polar plot of surface free energy for a crystal (a) and the Wulff construction based on it (b), and hence the void shape consisting of four facets joined by smoothly curved regions. 


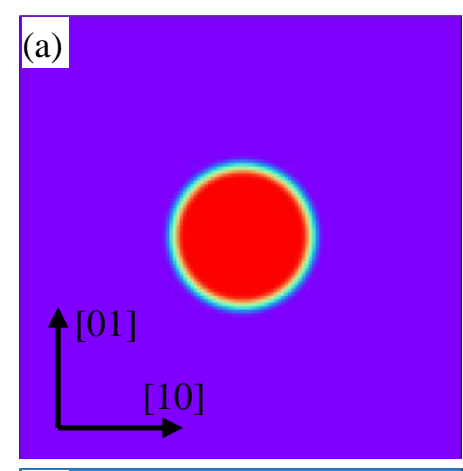

(d)
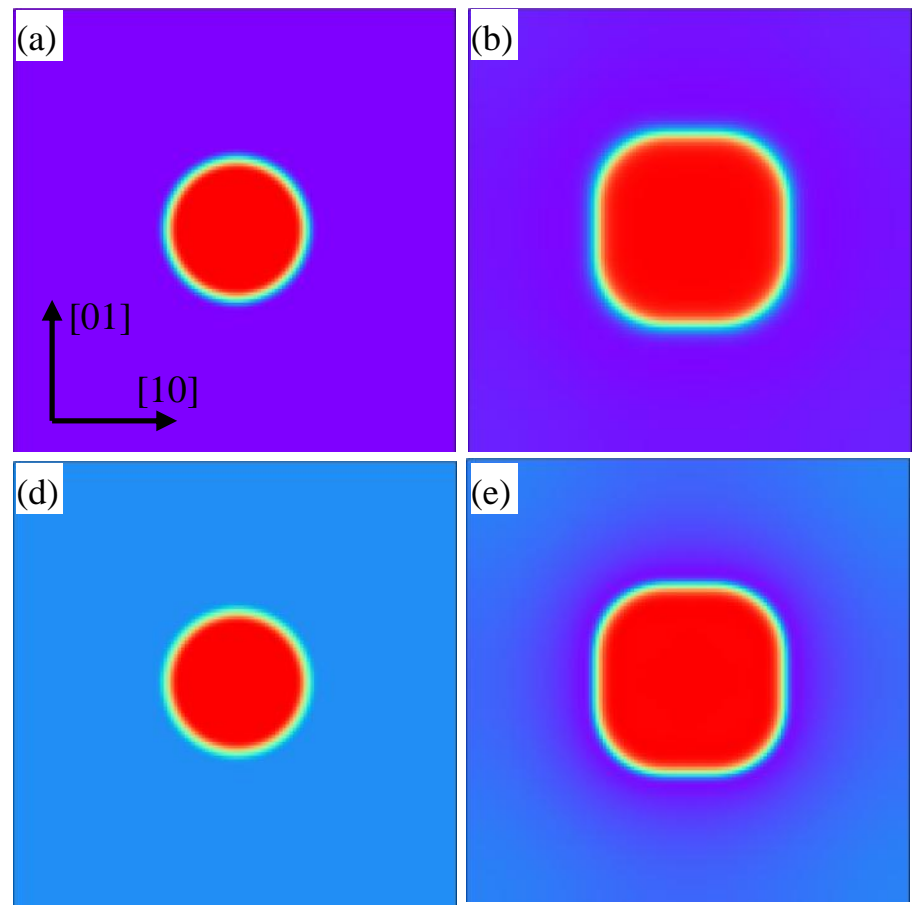

(e)
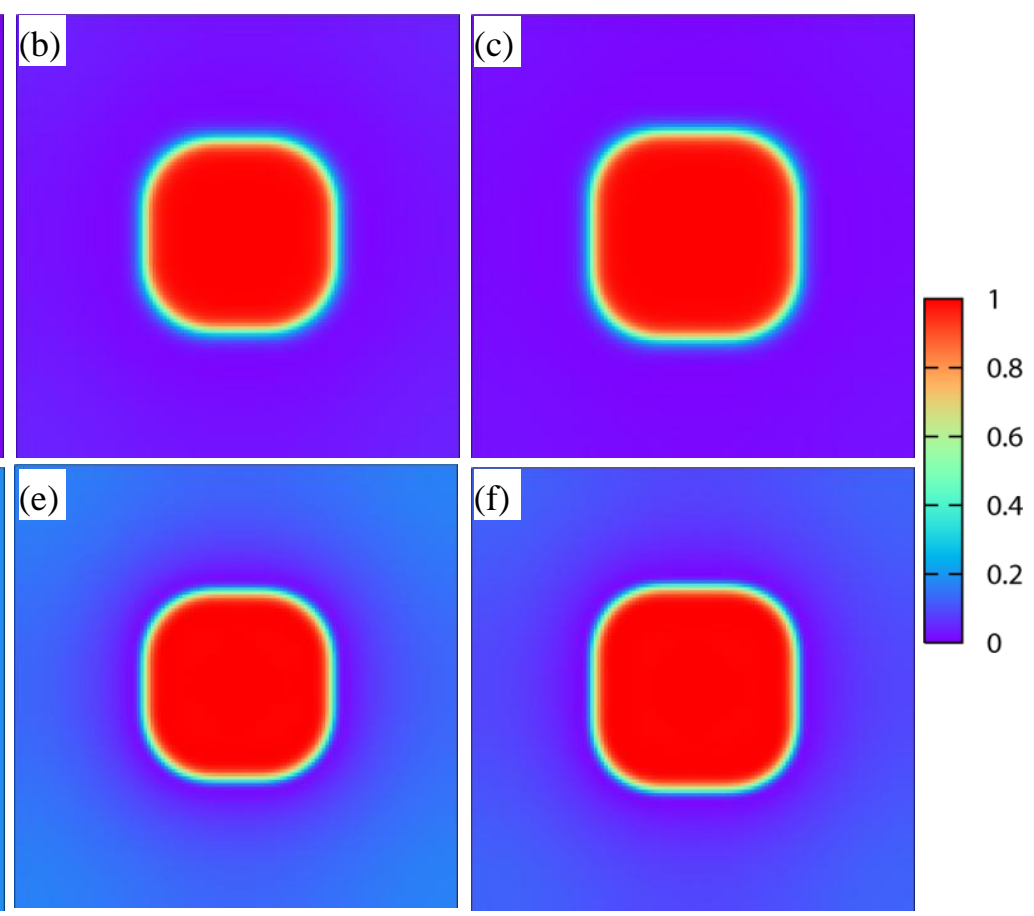

Fig.2 Effects of surface energy anisotropy on the void morphology evolutions of $\eta(\tilde{\mathbf{r}}, \tilde{t})$ and $c_{v}(\tilde{\mathbf{r}}, \tilde{t})$ with an initial supersaturation of vacancies: (a) $\eta(\tilde{\mathbf{r}}, 0)$, (b) $\eta(\tilde{\mathbf{r}}, 45)$, (c) $\eta(\tilde{\mathbf{r}}, 90)$, (d) $c_{v}(\tilde{\mathbf{r}}, 0),(\mathrm{e}) c_{v}(\tilde{\mathbf{r}}, 45),(\mathrm{f}) c_{v}(\tilde{\mathbf{r}}, 90)$. Inset in (a) shows a global coordinate $\left(\theta_{0}=0\right)$. 

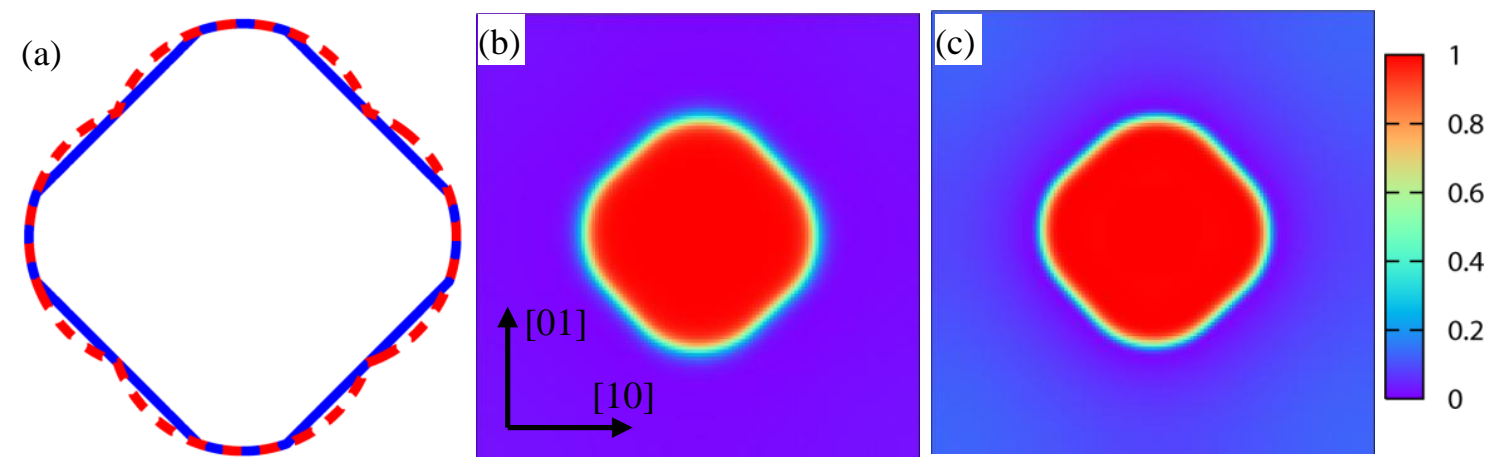

Fig.3 Effects of surface energy anisotropy on the morphology evolutions with orientation of $\theta_{0}=45^{\circ}$ in Eq. (11): (a) $\gamma$ plot (dashed red line) and inner envelope (full blue line) (b) $\eta(\tilde{\mathbf{r}}, 90)$ and (c) $c_{v}(\tilde{\mathbf{r}}, 90)$. Inset in (b) shows a global coordinate $\left(\theta_{0}=0\right)$. 

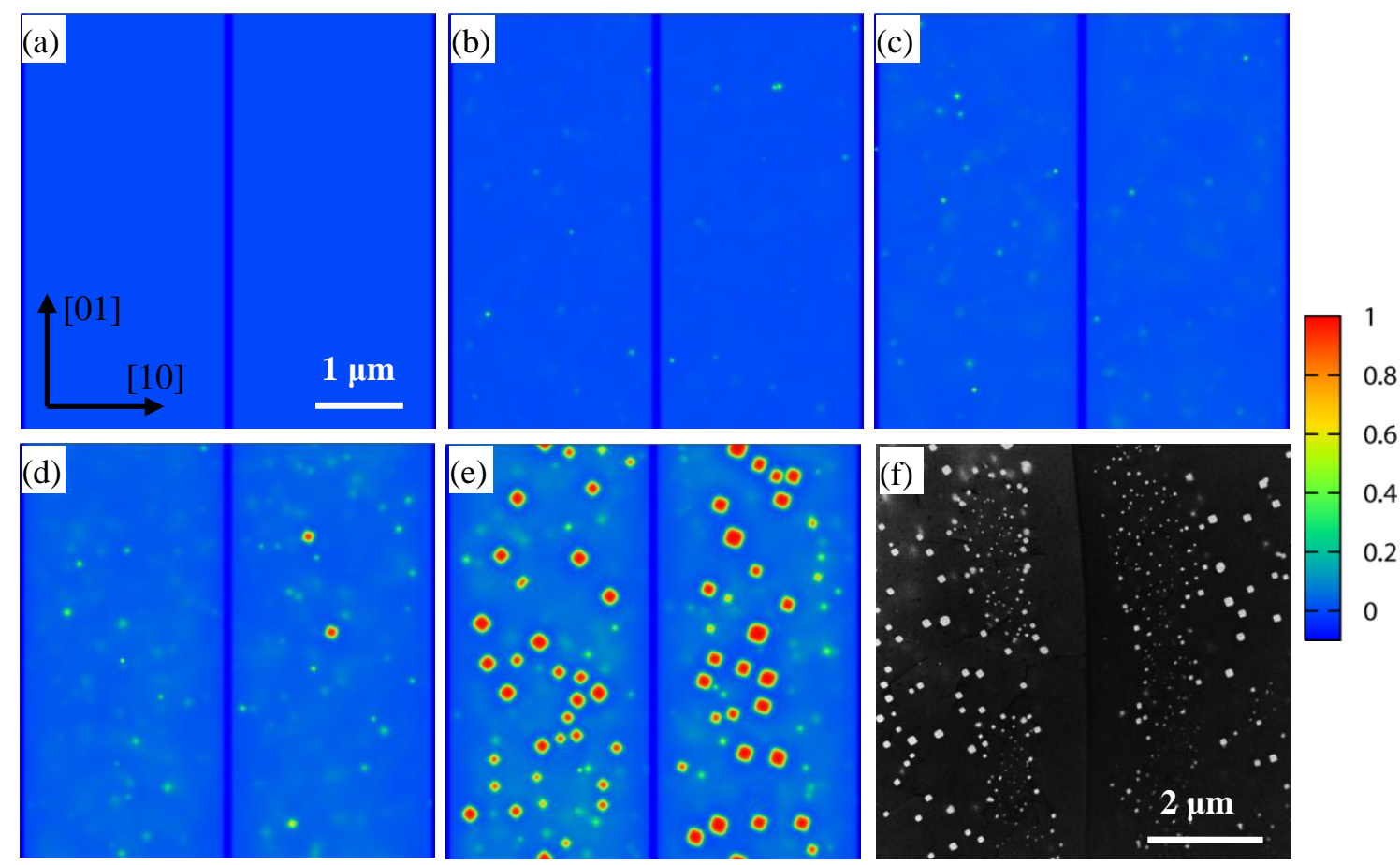

Fig.4 Void morphology evolutions in a bi-crystal structure under the effect of surface energy anisotropy for different grains: (a)0 dpa, (b) $0.09 \mathrm{dpa}$, (c) $0.18 \mathrm{dpa}$, (d) $0.27 \mathrm{dpa}$, (e) $0.36 \mathrm{dpa}$. This phenomenon has been observed in irradiated copper [11] as shown in (f) (picture taken from [11]). In all the simulations (a), (b), (c), (d) and (e), the crystalline orientation angles $\theta_{0}$ are set as $\theta_{0}=45^{\circ}$ for the left grain and $\theta_{0}=22.5^{\circ}$ for the right grain. Inset in (a) shows a global coordinate $\left(\theta_{0}=0\right)$. 

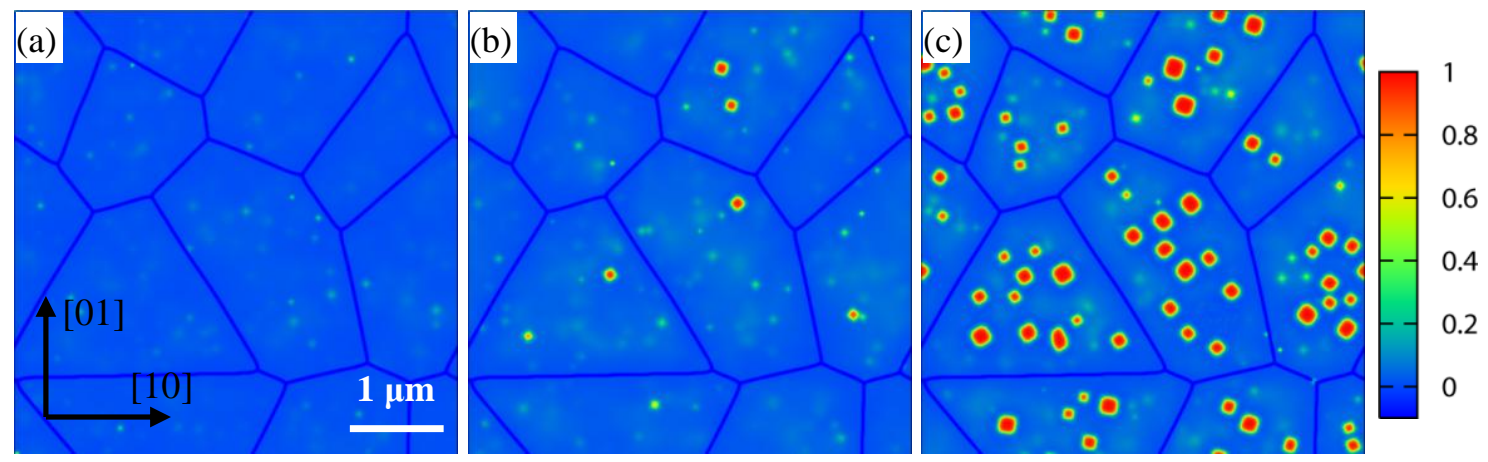

Fig.5 Void morphology evolutions in a polycrystalline grain structure under the effect of surface energy anisotropy and stochastic vacancy generation: (a) $0.15 \mathrm{dpa}$, (b) $0.225 \mathrm{dpa}$, (c) $0.3 \mathrm{dpa}$. Inset in (a) shows a global coordinate $\left(\theta_{0}=0\right)$. 